\title{
CHARACTERIZATION OF LOWER SEMICONTINUOUS CONVEX FUNCTIONS
}

\author{
R. CORREA, A. JOFRÉ, AND L. THIBAULT
}

(Communicated by William J. Davis)

\begin{abstract}
We prove that a lower semicontinuous function defined on a reflexive Banach space is convex if and only if its Clarke subdifferential is monotone.
\end{abstract}

\section{INTRODUCTION}

In [8] Poliquin showed that a lower semicontinuous function defined on $\mathbb{R}^{n}$ is convex if (and only if) its Clarke subdifferential is a monotone set-valued operator. For the particular case of a locally Lipschitzian function this fact had been remarked by Clarke [4, Proposition 2.2.9].

In this paper we extend this result to a lower semicontinuous function defined on a reflexive Banach space. The method used by Poliquin depends heavily on the notion of quadratic conjugate function that he introduces. In our work the use of the Moreau-Yosida proximal approximation allows us to prove the result more generally in a reflexive Banach space.

\section{DEFINITIONS AND PROPERTIES}

In this paper $E$ is a reflexive Banach space and we always consider lower semicontinuous functions from $E$ into $\mathbb{R} \cup\{+\infty\}$ that are not identically equal to $+\infty$.

It is known [5, Corollary 3, p. 167, Theorem 4, p. 32] that $E$ admits an equivalent Kadec norm, that is, a norm $\|\cdot\|$ for which a sequence $\left(y_{n}\right)$ in $E$ converges to $\bar{y}$ provided that $\left(y_{n}\right)$ is weakly convergent to $\bar{y}$ and $\left\|y_{n}\right\| \rightarrow\|\bar{y}\|$. In the sequel we assume that $E$ is endowed with such a norm.

In what follows we recall some notions and properties from nonsmooth analysis.

The Fenchel-subdifferential of a function $f$ at a point $a \in E$ such that $f(a) \in \mathbb{R}$ is the set

$$
\partial^{c} f(a)=\left\{x^{*} \in E^{*}: f(a+d)-f(a) \geq\left\langle x^{*}, d\right\rangle \text { for all } d \in E\right\}
$$

where $E^{*}$ denotes the topological dual of $E$ and $\langle\cdot, \cdot\rangle$ the bilinear form of the duality. For the properties of $\partial^{c} f$ see Moreau [7, Chapters 10-12] and

Received by the editors February 3, 1991.

1991 Mathematics Subject Classification. Primary 49A52; Secondary 49A51, 47H05.

Partially supported by Fondo Nacional de Ciencias. 
Rockafellar [9, Part 5]. The Clarke-subdifferential of a function $f$ at a point $a \in E$ such that $f(a) \in \mathbb{R}$ is the set

$$
\partial f(a)=\left\{x^{*} \in E^{*}: f^{\uparrow}(a ; d) \geq\left\langle x^{*}, d\right\rangle \text { for all } d \in E\right\}
$$

where

$$
f^{\uparrow}(a ; d)=\limsup _{\substack{x \rightarrow f^{a} \\ t \rightarrow 0^{+}}} \inf _{y \rightarrow d} \frac{f(x+t y)-f(x)}{t} .
$$

For the definition of limsupinf and the properties of $\partial f$ and $f^{\uparrow}$ see Clarke [4, Chapter 2] and Rockafellar [10]. We extend these two definitions by setting $\partial f(a)=\partial^{c} f(a)=\varnothing$ when $f(a)=+\infty$.

We recall now four properties of these subdifferentials.

2.1. Property. $\partial^{c} f(a) \subset \partial f(a)$.

2.2. Property. $\partial f$ is nonempty in a dense subset of the set where $f$ is finite. Moreover, when $f$ is locally Lipschitzian, $\partial f(x) \neq \varnothing$ for all $x \in E$.

2.3 Property. If $f$ is convex, then $\partial^{c} f(a)=\partial f(a)$.

We recall that a set-valued operator $T: E \rightarrow E^{*}$ is monotone if

$$
x^{*} \in T(x), z^{*} \in T(z) \Rightarrow\left\langle x^{*}-z^{*}, x-z\right\rangle \geq 0 .
$$

2.4. Property. If $f$ is convex, then the set-valued operator $\partial f$ is monotone.

Property 2.2 is due to Mc Linden [6], Property 2.4 follows immediately from Property 2.3 and the definition of $\partial^{c} f$. Our aim in this work is to show that the converse of Property 2.4 is also true in reflexive spaces.

One difficulty in the study of the converse of Property 2.4 is that we must work directly with the Clarke-subdifferential. In fact, the Fenchel-subdifferential is monotone for any function. This means that the monotonicity of these two subdifferential set-valued operators are not equivalent.

In our arguments we need the following intermediate subdifferential.

2.5. Definition. The Frechet-subdifferential of a function $f$ at a point $a \in E$ such that $f(a) \in \mathbb{R}$ is the set

$$
\partial^{F} f(a)=\left\{x^{*} \in E^{*}: \liminf _{y \rightarrow 0} \frac{f(a+y)-f(a)-\left\langle x^{*}, y\right\rangle}{\|y\|} \geq 0\right\} .
$$

2.6. Property. If $f$ is locally Lipschitzian, then

$$
\partial f(a)=\overline{\operatorname{co}} w^{*}-\limsup _{x \rightarrow a} \partial^{F} f(x)
$$

where $\overline{\operatorname{co}} T$ denotes the closed convex hull of the set $T \subset E^{*}$ and $w^{*}$-limsup $\sup _{x \rightarrow a} T(x)$ denotes the $w^{*}$ sequential upper limit of the sets $T(x) \subset$ $E^{*}$ in Kuratowski's sense.

For this equality and other properties of $\partial^{F} f$ see Borwein and Strojwas [3].

2.7. Property. $\partial^{c} f(a) \subset \partial^{F} f(a) \subset \partial f(a)$.

Properties 2.7 and 2.6 show that the monotonicity of $\partial f$ is equivalent to that of $\partial^{F} f$ when $f$ is a locally Lipschitzian function.

\section{THE CHARACTERIZATION}

In Lemma 3.2 we use the following approximate mean-value theorem recently proved by Zagrodny [10]. 
3.1. Approximate mean value theorem. Let $a, b \in E$ such that $f(a), f(b) \in$ $\mathbb{R}$. Then there exist $c \in] a, b]$, a sequence $\left(x_{k}\right)$ converging to $c$, and $x_{k}^{*} \in$ $\partial f\left(x_{k}\right)$ such that

$$
\frac{\|b-a\|}{\|c-a\|} \lim \sup \left\langle x_{k}^{*}, x_{k}-a\right\rangle \leq f(b)-f(a) .
$$

3.2. Lemma. If $\partial f$ is monotone, then $\partial f(x)=\partial^{c} f(x)$ for all $x \in E$.

Proof. Let $x \in E$. If $\partial f(x)=\varnothing$ the equality is evident. If $\partial f(x) \neq \varnothing$ then $f(x) \in \mathbb{R}$, and from the approximate mean value theorem for each $d \in E$ such that $f(x+d) \in \mathbb{R}$ there exist a sequence $v_{k} \rightarrow d$, a sequence $\left.\left.t_{k} \rightarrow t \in\right] 0,1\right]$, and $x_{k}^{*} \in \partial f\left(x+t_{k} v_{k}\right)$ such that

$$
f(x+d)-f(x) \geq t^{-1} \lim \sup \left\langle x_{k}^{*}, x+t_{k} v_{k}-x\right\rangle .
$$

Then, the monotonicity of $\partial f$ implies that for all $x^{*} \in \partial f(x)$

$$
f(x+d)-f(x) \geq t^{-1} \lim \sup \left\langle x^{*}, x+t_{k} v_{k}-x\right\rangle=\left\langle x^{*}, d\right\rangle .
$$

This shows that $x^{*} \in \partial^{c} f(x)$, that is, $\partial f(x) \subset \partial^{c} f(x)$. The equality follows from Property 2.1 .

It is easy to prove that if $\left\{x \in E: \partial^{c} f(x) \neq \varnothing\right\}=E$ then the function $f$ is convex. In fact let $x, y, z=\lambda x+(1-\lambda) y$ in $E$, and $z^{*} \in \partial^{c} f(z)$, then we have $f(x) \geq f(z)+\left\langle z^{*}, x-z\right\rangle$ and $f(y) \geq f(z)+\left\langle z^{*}, y-z\right\rangle$, which show that for $\lambda \in[0,1], \lambda f(x)+(1-\lambda) f(y) \geq f(z)$. This fact jointly with Lemma 3.2 show that if $\partial f$ is monotone and nonempty valued, then the function $f$ is convex. For this reason we will use a pointwise approximation $f_{\lambda}$ of $f$ such that its Clarke subdifferential is monotone and nonempty valued.

3.3. Definition. For any $\lambda>0$ the Moreau-Yosida proximal approximation of index $\lambda$ of a function $f$ is

$$
f_{\lambda}(x)=\inf _{y \in E}\left[f(y)+\frac{1}{2 \lambda}\|x-y\|^{2}\right] .
$$

3.4. Property. If $f$ is bounded from below by a continuous affine function, then $f_{\lambda}$ is finite and locally Lipschitzian.

3.5. Property. If $f$ is bounded from below by a continuous affine function, then $f=\sup _{\lambda>0} f_{\lambda}$.

For the proof of Properties 3.4 and 3.5 see [1].

The monotonicity of $\partial f$ implies, by Lemma 3.2 and Property 2.2 , that $f$ is bounded from below by a continuous affine function. Then, Properties 2.2 and 3.4 ensure that $\left\{x \in E: \partial f_{\lambda}(x) \neq \varnothing\right\}=E$, hence if we can prove the monotonicity of $\partial f_{\lambda}$ we will obtain, by Lemma 3.2 again, the convexity of $f_{\lambda}$. After this, the convexity of $f$ will be an immediate consequence of Property 3.5.

3.6. Lemma. If the convolution

$$
(f \square g)(a)=\inf _{y \in E}[f(y)+g(a-y)]
$$

is exact, that is, if the above infimum is attained at $\bar{y}$, then

$$
\partial^{F}(f \square g)(a) \subset \partial^{F} f(\bar{y}) \cap \partial^{F} g(a-\bar{y}) .
$$


Proof. Let $x^{*} \in \partial^{F}(f \square g)(a)$ and $y \in E$, then

$$
\begin{aligned}
f(\bar{y}+y)-f(\bar{y})-\left\langle x^{*}, y\right\rangle & =f(\bar{y}+y)+g(a-\bar{y})-f(\bar{y})-g(a-\bar{y})-\left\langle x^{*}, y\right\rangle \\
& \geq(f \square g)(a+y)-(f \square g)(a)-\left\langle x^{*}, y\right\rangle .
\end{aligned}
$$

Dividing by $\|y\|$ and taking the lower limit as $\|y\| \rightarrow 0$, we obtain that $x^{*} \in \partial^{F} f(\bar{y})$. Analogously it can be shown that $x^{*} \in \partial^{F} g(a-\bar{y})$.

3.7. Lemma. If $\partial f$ is monotone then $\partial f_{\lambda}$ is monotone.

Proof. (1) In order to use the inclusion in Lemma 3.6 we will first show, following the technique in the proof of Theorem 11 in [2], that if $\partial^{F} f_{\lambda}(a) \neq \varnothing$, then there exists $\bar{y}$ such that $f_{\lambda}(a)=f(\bar{y})+(1 / 2 \lambda)\|a-\bar{y}\|^{2}$.

Fix a sequence $\left(t_{n}\right)$ of positive number converging to 0 and find a sequence $\left(y_{n}^{\prime}\right)$ such that

$$
f\left(y_{n}^{\prime}\right)+\frac{1}{2 \lambda}\left\|a-y_{n}^{\prime}\right\|^{2} \leq f_{\lambda}(a)+t_{n}^{2} .
$$

From Lemma 3.2 and Property 2.2 we know that the function $f$ is bounded from below by a continuous affine function. Then the sequence $\left(y_{n}^{\prime}\right)$ must be bounded and there exists a subsequence $\left(y_{n}\right)$ converging weakly to some $\bar{y}$ and such that $\left\|a-y_{n}\right\| \rightarrow \alpha$.

We will prove that $\|\bar{y}-a\|=\alpha$ and, since \|\| is Kadec, we will conclude that $\left(y_{n}\right)$ strongly converges to $\bar{y}$. Then, taking the lower limit in (A) the desired equality will be obtained.

The inequality $\|a-\bar{y}\| \leq \alpha$ is a consequence of the weak lower semicontinuity of the norm.

Let us prove that $\|a-\bar{y}\| \geq \alpha$.

For any $\varepsilon>0, x^{*} \in \partial^{F} f_{\lambda}(a)$, and $n$ large enough, we have

$$
\begin{aligned}
\left\langle x^{*},\right. & \left.y_{n}-a\right\rangle \\
& \leq t_{n}^{-1}\left[f_{\lambda}\left(a-t_{n}\left(a-y_{n}\right)\right)-f_{\lambda}(a)\right]+\varepsilon \\
& \leq t_{n}^{-1}\left[f\left(y_{n}\right)+\frac{1}{2 \lambda}\left\|\left(1-t_{n}\right)\left(a-y_{n}\right)\right\|^{2}-f\left(y_{n}\right)-\frac{1}{2 \lambda}\left\|a-y_{n}\right\|^{2}+t_{n}^{2}\right]+\varepsilon \\
& =-\frac{1}{\lambda}\left\|a-y_{n}\right\|^{2}+\frac{t_{n}}{2 \lambda}\left\|a-y_{n}\right\|^{2}+t_{n}+\varepsilon,
\end{aligned}
$$

and taking the limit, we obtain for all $\varepsilon>0$

$$
\left\langle x^{*}, \bar{y}-a\right\rangle \leq-\frac{1}{\lambda} \alpha^{2}+\varepsilon
$$

then

$$
\frac{\alpha^{2}}{\lambda} \leq\left\|x^{*}\right\|\|a-\bar{y}\| .
$$

Analogously, for any $\varepsilon>0, x \in E, x^{*} \in \partial^{F} f_{\lambda}(a)$, and $n$ large enough, we have

$$
\begin{aligned}
\left\langle x^{*}, x\right\rangle & \leq t_{n}^{-1}\left[f_{\lambda}\left(a+t_{n} x\right)-f_{\lambda}(a)\right]+\varepsilon \\
& \leq t_{n}^{-1}\left[f\left(y_{n}\right)+\frac{1}{2 \lambda}\left\|a-y_{n}+t_{n} x\right\|^{2}-f\left(y_{n}\right)-\frac{1}{2 \lambda}\left\|a-y_{n}\right\|^{2}+t_{n}^{2}\right]+\varepsilon \\
& \leq \frac{1}{\lambda}\left\|a-y_{n}\right\|\|x\|+\frac{t_{n}}{2 \lambda}\|x\|^{2}+t_{n}+\varepsilon,
\end{aligned}
$$


and taking the limit, we obtain for all $\varepsilon>0$ and $x \in E$

$$
\left\langle x^{*}, x\right\rangle \leq \frac{1}{\lambda} \alpha\|x\|+\varepsilon
$$

then

$$
\left\|x^{*}\right\| \leq \frac{\alpha}{\lambda}
$$

From (B) and (C) we obtain that $\|a-\bar{y}\| \geq \alpha$.

(2) From Property 2.7 , the monotonicity of $\partial f$ implies that of $\partial^{F} f$. Let us prove now that $\partial^{F} f_{\lambda}$ is monotone.

Let $x^{*} \in \partial^{F} f_{\lambda}(x)$ and $y^{*} \in \partial^{F} f_{\lambda}(y)$. From Lemma 3.6 and the first part of the proof of the lemma there exist $\bar{x}, \bar{y}$ such that

$$
\partial^{F} f_{\lambda}(x) \subset \partial^{F} f(\bar{x}) \cap \partial^{F} g(x-\bar{x}) \text { and } \partial^{F} f_{\lambda}(y) \subset \partial^{F} f(\bar{y}) \cap \partial^{F} g(y-\bar{y})
$$

where $g=(1 / 2 \lambda)\|\cdot\|^{2}$. Then

$$
\left\langle x^{*}-y^{*}, x-y\right\rangle=\left\langle x^{*}-y^{*}, \bar{x}-\bar{y}\right\rangle+\left\langle x^{*}-y^{*},(x-\bar{x})-(y-\bar{y})\right\rangle \geq 0 .
$$

The equivalence between the monotonicity of $\partial^{F} f_{\lambda}$ and $\partial f_{\lambda}$ (Property 2.6) completes the proof.

3.8. Theorem. If $\partial f$ is monotone then $f$ is convex.

Proof. From Lemma 3.7 we know that the monotonicity of $\partial f$ implies that of $\partial f_{\lambda}$. Then, from Lemma 3.2 we see that $\partial f_{\lambda}=\partial^{c} f_{\lambda}$ and, since $\partial f_{\lambda}(x)$ is nonempty for any $x \in E$, we conclude that $f_{\lambda}$ must be a convex function.

The convexity of $f$ is a consequence of Property 3.5.

Examples. The following examples (a) and (b) show respectively that the lower semicontinuity of $f$ and the monotonicity of $\partial f$ (on domain of $\partial f$ ) are necessary.

$$
f(x)=\left\{\begin{array}{ll}
0, & x \neq 0, \\
1, & x=0,
\end{array} \quad \partial f(x)= \begin{cases}0, & x \neq 0 \\
\varnothing, & x=0\end{cases}\right.
$$

hence $\partial f$ is monotone but $f$ is not convex.

$$
f(x)=\left\{\begin{array}{ll}
0, & x \neq 0, \\
-1, & x=0,
\end{array} \quad \partial f(x)= \begin{cases}0, & x \neq 0, \\
\mathbb{R}, & x=0\end{cases}\right.
$$

hence $f$ is lower semicontinuity, $\partial f$ is monotone on $\mathbb{R} \backslash\{0\}$ dense in domain of $\partial f$, and $f$ is not convex.

3.9. Remark. (1) Notice that the proof is simpler whenever $E$ is finite dimensional. Indeed in this case the function $f+(1 / 2 \lambda)\|x-\cdot\|^{2}$ is inf-compact and hence the first part of the proof of Lemma 3.7 is unnecessary.

(2) The Hadamard subdifferential could be used instead of the Frechet subdifferential.

3.10 Remark. Symmetric arguments allow us to prove that if $-\partial f$ is monotone then $f$ is concave; in this sense, we recall that in general $-\partial f$ is not equal to $\partial(-f)$. 


\section{REFERENCES}

1. H. Attouch, Variational convergence for functions and operators, Pitman, London, 1984.

2. J. M. Borwein and J. R. Giles, The proximal normal formula in Banach space, Trans. Amer. Math. Soc. 302 (1987), 371-381.

3. J. M. Borwein and H. M. Strojwas, Proximal analysis and boundaries of closed sets in Banach space II: Applications, Canad. J. Math. 39 (1987), 428-472.

4. F. H. Clarke, Optimization and nonsmooth analysis, Wiley, New York, 1983.

5. J. Diestel, Geometry of Banach spaces-selected topics, Lecture Notes in Math., vol. 485, Springer-Verlag, New York, 1975.

6. L. Mc. Linden, On application of Ekeland's theorem to minimax problems, Nonlinear Anal. Theory Methods Appl. 6 (1982), 189-196.

7. J. J. Moreau, Fonctionelles convexes, Lecture Notes, Séminaire Equations aux dérivées partielles, Collège de France, 1966.

8. R. A. Poliquin, Subgradient monotonicity and convex functions, Nonlinear Anal. Theory Methods Appl. 14 (1990), 305-317.

9. R. T. Rockafellar, Convex analysis, Princeton Univ. Press, Princeton, NJ, 1970.

10. _ Generalized directional derivatives and subgradients of nonconvex functions, Canad. J. Math. 32 (1980), 257-280.

11. D. Zagrodny, Approximate mean value theorem for upper subderivatives, Nonlinear Anal. Theory Methods Appl. 12 (1988), 1413-1438.

(R. Correa and A. Jofré) Departamento de Ingenierfa Matemática, Universidad de Chile, Casilla 170/3-Correo 3, Santiago, Chile

(L. Thibault) Defartement de Mathématiques, Université de PaU, Av. De L'Université, 64000 Pau, France 